\title{
БАЗЫ ДАННЫХ В СТРУКТУРЕ ПРОГРАММНО-АППАРАТНОГО КОМПЛЕКСА, ПОСРЕДСТВОМ КОТОРОГО ВЫПОЛНЯЕТСЯ УПРАВЛЕНИЕ СИСТЕМЫ «УМНЫЙ ДОМ»
}

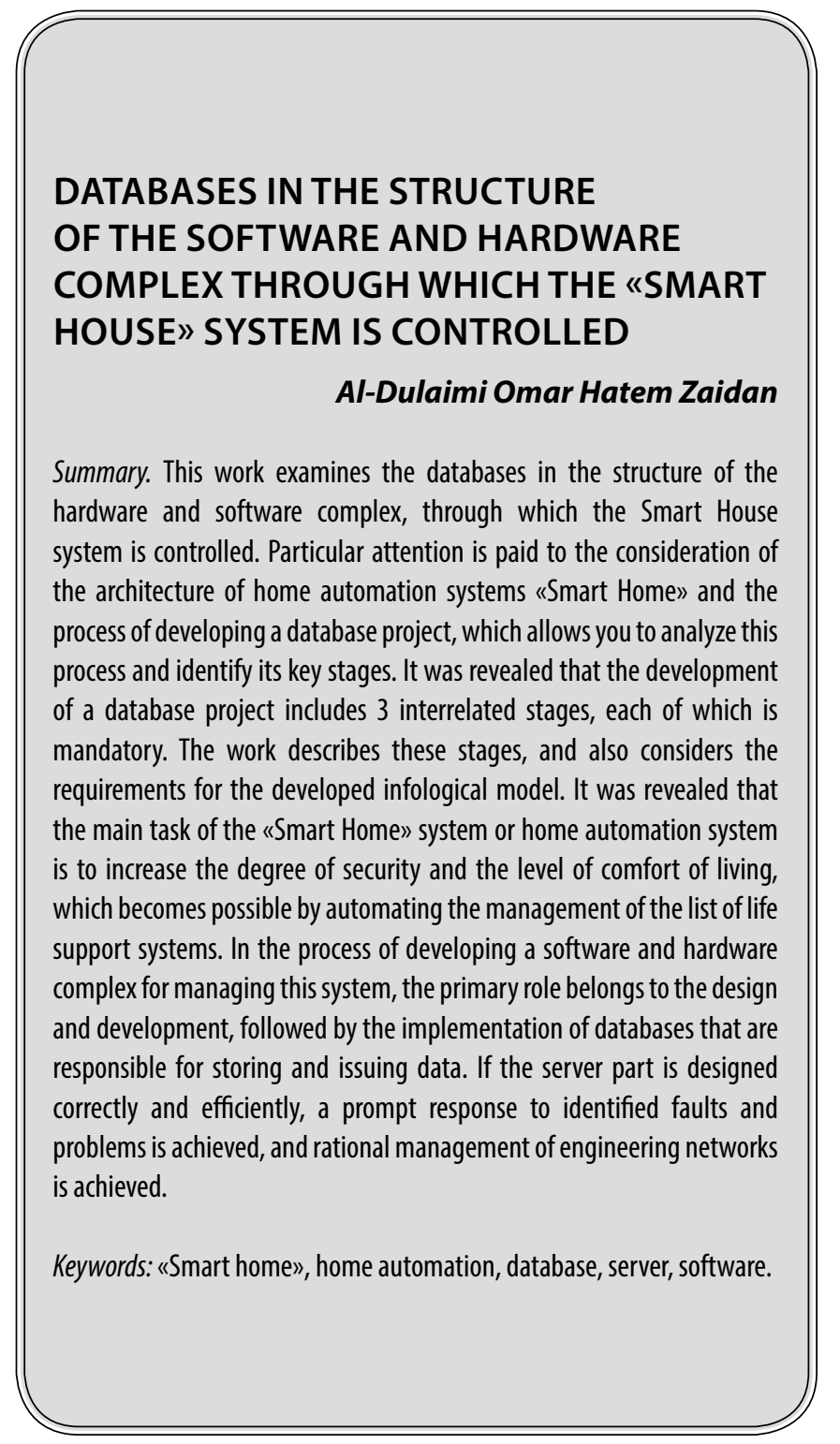

\section{Введение}

истема автоматизации для дома, известная более как система «Умный дом», ее разработка и исполнение представляет собой современный вектор промышленной автоматизации, вызывающий высокий интерес как у исследователей, так и у потребителей [1, 2, 3]. Если рассматривать данную систему с позиции инженерного устройства, это комплект специализированных

\author{
Аль-дулаими Омар Хатем Заидан \\ Аспирант, Воронежский государственный \\ технический университет \\ omarhatem82@gmail.com
}

Аннотация. В данной работе рассматриваются базы данных в структуре программно-аппаратного комплекса, посредством которого выполняется управление системы «Умный дом». Особое внимание в работе уделяется рассмотрение архитектуры систем домашней автоматизации «Умный дом» и процессу разработки проекта базы данных, что позволяет проанализировать данный процесс, выявить его ключевые этапы. Было выявлено, что разработка проекта базы данных включает в себя 3 взаимосвязанных этапа, каждый из которых является обязательным. В работе описываются данные этапы, а также рассматриваются требования для разрабатываемой инфологической модели. Было выявлено, что главной задачей системы «Умный дом» или системы домашней автоматизации является увеличение степени безопасности и уровня комфорта проживания, которое становится возможным за счет автоматизации управления перечнем систем жизнеобеспечения. В процессе разработки программно-аппаратного комплекса для осуществления управления данной системой первостепенная роль принадлежит проектированию и разработке с последующим выполнением баз данных, которые ответственны за хранение и выдачу данных. Если серверная часть разработана корректно и эффективно, достигается оперативное реагирование на выявленные неполадки и проблемы, а также достигается рациональное управление инженерными сетями.

Ключевые слова: «Умный дом», домашняя автоматизация, база данных, сервер, программное обеспечение.

технических средств и программного обеспечения, основной задачей которых является формирование интегрированной системы автоматизации разноуровневых инженерных подсистемы, адаптируемых под пользовательские потребности и управляемые искусственным интеллектом [4]. Наибольшего внимания в структуре системы «Умный дом» заслуживает серверная часть, так как она ответственна за оперативный сбор, обработку и последующую передачу данных. 


\section{Архитектура систем «Умный $\triangle О м »$}

Архитектура системы «Умный дом» включает в себя 3 основные части: клиентскую, аппаратную и серверную.

Серверная часть состоит из 2 уровней, логического и интеллектуального. Логический уровень серверной части ответственен за сбор, анализ и распознавание, дальнейшее хранение и выполнение сравнительного анализа получаемых от контроллеров данных. Устройства логического уровня регистрируют отклонения от выработанного рабочего режима, в ситуациях выявления критических показателей клиенту подается сигнал о выявленных неполадках, также логический уровень осуществляет запуск комплекса управленческих действий, направленных на своевременное предотвращение аварийного отключения оборудования и всей системы в целом. В физическом виде логический уровень состоит из веб-сервера и контроллеров.

Интеллектуальный уровень серверной части состоит из распределенной базы данных, которая, в свою очередь, состоит из тематической и инструментальной частей. Инструментальная часть базы данных интеллектуального уровня серверной части система «Умный дом» содержит набор правил, регламентирующих процесс управления программным и аппаратным обеспечением всей системы. Тематическая часть базы данных интеллектуального уровня серверной части система «Умный дом» включает набор правил, разработанных предметным специалистом с целью реализации технологических инструкций, которые относятся к системе управления.

Таким образом, сервер и база данных представляю собой основополагающие элементы серверной части. База данных содержит произведенные настройки, информацию о всей системе, ряд показаний, которые предоставляются при возникновении необходимости. Сервер - это интерфейс для настройки системы, наблюдения за ее функционированием и управления [5]. Сервер делает схемы использования ресурсов более рациональными, для этого он связывает устройства помещений с пользователей.

Схема взаимодействия подсистем серверной части выглядит следующим образом:

1. Устройства осуществляют сбор данных с использующихся датчиков.

2. Собранные данные отправляются на сервер.

3. Веб-сервер осуществляет обработку поступающих данных.

4. Веб-сервер регистрирует данные, вводит их в базу данных.

5. Пользователь делает запрос на те или иные данные из базы данных.
6. Веб-сервер предоставляет необходимые данные $[6,7]$.

Если рассматривать базу данных, она имеет собственную структуру, представленную 4 уровнями иерархии понятий:

- Первый уровень - системный элемент. Это прибор, функционирование которого необходимо автоматизировать.

- Второй уровень - устройство.

- Третий уровень - актуатор. Это исполнитель, который выполняет автоматизацию требуемой функции.

- Четвертый уровень - воздействие активации. Это определенное действие, которое необходимо для стимулирования актуатора, его выхода из текущего состояния [8].

Перечисленные уровни позволяют увеличивать системную структуру и показатели функциональности, что упрощает настройки делает всю систему универсальной.

На практике зачастую база данных являются комплексом табличных коллекций, организованных для обеспечения рационального хранения сведений. Таблицы взаимосвязаны друг с другом и классифицируются на 3 области.

Первой областью является пользовательская область, которая задает системную роль пользователя. Пользовательская область содержит таблицы для хранения и использования следующих пользовательских сведений: системные роли, идентификатор, имя, телефонный номер, адрес электронной почты, число попыток неуспешных входов и так далее.

Второй областью является область локации, которая представлена перечнем таблиц для хранения и использования сведений о месторасположения объекта: названий государства, города, улицы, точного адреса объекта со специализированным идентификатором, а также с пользовательским идентификатором.

Третьей областью является область дома. Это таблицы для хранения и использования данных о пользовательских объектах, а также об установленных на территории пользовательских объектов контроллеров и датчиков. В таблицах области дома содержатся такие сведения как специализированный идентификатор объекта, полное название, адрес, характеристики объекта (сколько этажей, сколько комнат, габариты комнат, количество окон и дверей, наличие системы «теплый пол», наличие и количество источников воды, электроэнергии, газа), сведения о правах доступа к объекту, информация о количестве детей и домашних питомцев в объ- 
екте, информация о контроллерах в комнате, сведения об устройствах, которые находятся на контроллере [9].

\section{Разработка проекта базы манных}

Система «Умный дом» является сложной предметной областью, для которых затруднительно построить концептуальную схему базы данных единовременно, необходимо разбить концептуальный этап на 2 уровня: даталогический и инфологический. При этом на первом уровне база данных описывается в соответствии с используемым языком описания система управления базы данных, а на втором уровне - в виде, который не зависит от используемой системы управления базой данных.

Разработка проекта базы данных включает в себя 3 взаимосвязанных этапа, каждый из которых является обязательным:

1. Инфологическое проектирование. На этом этапе выполняется анализ программного обеспечения.

2. Логическое проектирование. На этом этапе осуществляется формирование общей информационной модели программного обеспечения.

3. Физическое проектирование. На этом этапе разработанная логическая модель базы данных переносится в среду системы управления базой данных [10].

Важно учитывать, что разрабатываемая инфологическая модель должна соответствовать следующим требованиям:

- Средства модели должны описывать явления, предметы и объекты программного обеспечения, а также явления, предметы и объекты, которые применяются для решения прикладных задач, сюда же относятся отношения между перечисленными составляющими.

- Модельные средства должны описывать ограничения целостности информации, которые установлены программным обеспечением.

- Модельные средства устанавливают объемные характеристики данных, которые необходимы для разработки проектов базы данных.

- Ядро модельных понятий приближено к ядру понятий алгоритмов прикладных задач и лексическому аппарату специалистов программного обеспечения.

- В инфологической модели предусмотрены специализированные методы, задачей которых является объединение описаний прикладных задач и фрагментов программного обеспечения в единое описание полного программного обеспечения.

- Модельные средства характеризуют процесс эффективного разрешения прикладных задач с ука- занием операций, которые осуществляются над информацией, а также частоты их реализации.

- В инфологической модели предусмотрены способы и методы передачи, отображения единого полного описания программного обеспечения в последующие модели используемых систем управления баз данных.

Инфологическое проектирование состоит из исследования программного обеспечения и его информационной структуры, а также из обнаружения фрагментов, которые могут быть описаны, смоделированы и из интеграции собранных преставлений. Инфологическая модель, которая является семантической стороной программного обеспечения модели базы данных, необходимо сделать формализованное описание программного обеспечения с целью предоставления возможности понимания всеми участниками процесса, а не только специалистами базы данных. Данное описание должно соответствовать таким критериям как емкость и обобщенность, отсутствие привязки к определенной системе управления базой данных.

В процессе разработки модели и логической структуры базы данных инфологическая модель преобразуется в модель данных, которая поддерживается используемой системой управления базой данных, а далее осуществляется проверка адекватности модели относительно отображения программного обеспечения. Для любого используемого программного обеспечения можно применять проектные решения, при этом сделав отображение в даталогической модели. Чтобы отобразить инфологическую модель программного обеспечения «Умного дома» в даталогической структуре базы данных для определённой системы управления базы данных, необходимо применять инерационный процесс, подразумевающий прохождение 3 взаимосвязанных этапов:

1. Построение первоначальной логической структуры базы данных, которая обеспечивала бы максимально возможное сокращение объемов памяти в процессе сбора и дальнейшего хранения информации, различных данных.

2. Выбор методов физического обеспечения базы данных для построенной логической структуры базы данных, которые обеспечивали бы необходимое среднее и максимально возможное время решения прикладных задач с минимальными потерями памяти.

3. Модификация разработанной логической структуры базы данных, при условии отсутствия возможности найти оптимальные способы физической организации базы данных.

Физическая структура базы данных выбирается на основании унифицированной математической модели, при этом производительность служит главной целевой 
функцией. Модель в данном случае предусматривает разработку системы специализированных ограничений, учитывающую наибольшие значения допустимых временных и объемных характеристик, а также модель демонстрирует связи между структурными параметрами и несет ответственность за выполнение механизмов обеспечения надежной защиты всей системы.

\section{Зак^ючение}

Главной задачей системы «Умный дом» или системы домашней автоматизации является увеличение степени безопасности и уровня комфорта проживания, которое становится возможным за счет автоматизации управления перечнем систем жизнеобеспечения. В процессе разработки программно-аппаратного комплекса для осуществления управления данной системой первостепенная роль принадлежит проектированию и разработке с последующим выполнением баз данных, которые ответственны за хранение и выдачу данных. Если серверная часть разработана корректно и эффективно, достигается оперативное реагирование на выявленные неполадки и проблемы, а также достигается рациональное управление инженерными сетями.

\section{ЛИТЕРАТУРЫ}

1. Габдрахманова А.М., Султанович Е.Д. Рынок умного дома // Актуальные проблемы авиации и космонавтики. 2019. № . URL: https://cyberleninka.ru/ article/n/rynok-umnogo-doma (дата обращения: 13.10.2021).

2. Аверин А.И. Интеллектуальное управление домом. «Умный дом» // European science. 2015. № 4 (5). URL: https://cyberleninka.ru/article/n/intellektualnoeupravlenie-domom-umnyy-dom (дата обращения: 13.10.2021).

3. Кузяшев А.Н., Смолин А.Е. Интернет вещей, умный дом и умные города // Эпоха науки. 2021. № 25. URL: https://cyberleninka.ru/article/n/internet-vescheyumnyy-dom-i-umnye-goroda (дата обращения: 13.10.2021).

4. Байгозин Д.В., Первухин Д.Н., Захарова Г.Б. Разработка принципов интеллектуального управления инженерным оборудованием в системе «Умный дом» // Известия Томского политехнического университета. Инжиниринг георесурсов. — 2008.— Т. 313, № 5.— С. 168-172.

5. К Крамчанинов С.С., Черкесова Л.В. Общие вопросы проектирования программного обеспечения для системы домашней автоматизации «Умный загородный дом» // Academy. - 2018. - Т. 1, № 6 (33). - С. 18-22.

6. Михайлов Л.Ю. Разработка серверной части информационной системы «Умный дом»: выпуск. квалиф. работа: 02.03.03 / Л.Ю. Михайлов; Тольят. гос. унт.- Тольятти, 2016. - 65 с.

7. Ц Цой В.Г. Проектирование информационной системы «Умный дом»: выпуск. квалиф. работа: 09.03.03 / В.Г. Цой; Томский политех. ун-т.— Томск, 2017. $105 \mathrm{C}$.

8. Карпук А.А. Методология проектирования баз данных сложных систем // Банковская система: устойчивость и перспективы развития. Сборник научных статей пятой международной научно-практической конференции по вопросам банковской экономики, посвящённой 70-летию банковского образования на Полесье. Редколлегия: К.К. Шебеко [и др.]. — 2014. — С. 283-287.

9. Проектирование информационных систем: учебно-методическое пособие / Сост. Шамсутдинов Т.Ф. Казань: КГАСУ, 2018. — 110 c.

10. Яковина И.Н., Ногай Д.А., Собянин М.А., Волков А.А., Федрак А.М. Подход к разработке системы домашней автоматизации // Автоматика и программная инженерия. — 2015.— № 4 (14).—C. 46-53.

() Аль-дулаими Омар Хатем Заидан ( omarhatem82@gmail.com ). Журнал «Современная наука: актуальные проблемы теории и практики» 